\title{
OPTIMAL SELECTION OF THRESHOLD LEVELS AND WAVELET FILTERS FOR HIGH QUALITY ECG SIGNAL COMPRESSION
}

\section{Sabah Mohamed Ahmed}

Department of Electrical Engineering, Faculty of Engineering, Assiut University, Egypt (sabahma@yahoo.com)

(Received July 12, 2008 Accepted August 26, 2008)

Although most of the theoretical and implementation aspects of wavelet based algorithms in ElectroCardioGram (ECG) signal compression are well studied, many issues related to the choice of wavelet filters and threshold levels selection remain unresolved. The utilization of optimal mother wavelet will lead to localization and maximization of wavelet coefficients' values in wavelet domain. This paper presents an ECG compressor based on the optimal selection of wavelet filters and threshold levels in different subbands that achieve maximum data volume reduction while guaranteeing reconstruction quality. The proposed algorithm starts by segmenting the ECG signal into frames; where each frame is decomposed into $m$ subbands through optimized wavelet filters. The resulting wavelet coefficients are threshold and those having absolute values below specified threshold levels in all subands are deleted and the remaining coefficients are appropriately encoded with a modified version of the run-length coding scheme. The threshold levels to use, before encoding, are adjusted in an optimum manner, until predefined compression ratio and signal quality are achieved. Extensive experimental tests were made by applying the algorithm to ECG records from the MIT-BIH Arrhythmia Database [1]. The compression ratio (CR), the percent root-mean-square difference (PRD) and the zero-mean percent root-mean-square difference $\left(P R D_{1}\right)$ measures are used for measuring the algorithm performance (high CR with excellent reconstruction quality). From the obtained results, it can be deduced that the performance of the optimized signal dependent wavelet outperforms that of Daubechies and Coiflet standard wavelets. However, the computational complexity of the proposed technique is the price paid for the improvement in the compression performance measures. Finally, it should be noted that the proposed method is flexible in controlling the quality of the reconstructed signals and the volume of the compressed signals by establishing target PRD and CR a priori respectively.

KEY-WORDS: ECG signal compression; Discrete wavelet transform; Coding; Thresholding.

\section{INTRODUCTION}

Transmission techniques of biomedical signals through communication channels are currently an important issue in many applications related to clinical practice [8]-[9]. 
These techniques can allow experts to make a remote assessment of the information carried by the signals, in a very cost-effective way. However, in many situations this process leads to a large volume of information. The necessity of efficient data compression methods for biomedical signals is currently widely recognized. In electrocardiography, various channels are recorded during several hours, resulting in a great demand of storage capacity or channel bandwidth. This situation demands the use of efficient data compression systems. Although storage space is currently relatively cheap, electronic ECG archives could easily become extremely large and expensive. Moreover, sending ECG recordings through mobile networks would benefit from low bandwidth demands. Several examples of ECG compression algorithms have been described in the literature with compression ratios ranging approximately from 2:1 up to 50:1 [2]-[14]. These techniques can be categorized into: direct time-domain techniques; transformed frequency-domain techniques and parameters optimization techniques. Most time-domain techniques are based on the idea of extracting a subset of significant signal samples to represent the original signal. The key to a successful algorithm is the development of a good rule for determining the most significant samples. In this case, signal reconstruction is based on interpolating this subset of samples. The ECG time-domain techniques are, all in common, based on heuristics in the sample selection process. This generally makes them fast, but they all suffer from sub-optimality [2]. The second category of the ECG compression techniques utilizes the spectral and energy distributions of the signal by means of some transformation. This category includes traditional transform coding techniques applied to ECG signals such as the Karhunen-Loève transform, Fourier transform, cosine transform and wavelet transform (WT) [3]-[7]. Wavelet-based ECG compression methods have been proved to perform well [3], [8]-[9]. As reported in [9], the ability of WT to separate out pertinent signal components has led to a number of wavelet-based techniques which supersede those based on traditional Fourier methods. The discrete wavelet transform (DWT) has interesting mathematics and fits in with standard signal filtering and encoding methodologies. It produces few coefficients, and the user does not have to worry about losing energy during the transform process or its inverse. More recently, many interesting optimization based ECG compression methods, third category, have been developed [10]-[14]. The goal of most of these methods is to minimize the reconstruction error given a bound on the number of samples to be extracted [15] or the quality of the reconstructed signal to be achieved [4], [16].

The vast majority of the above mentioned methods do not permit perfect reconstruction of the original signals. In fact; there is no automatic way to assure that the distortion in the reconstructed signal will not affect clinical important features of the ECG. To guarantee the preservation of the clinical diagnostic features of the reconstructed ECG signals both the wavelet filters' parameters and the threshold levels in all subbands should be selected carefully. The aim of this study is to present ECG compression technique that achieves maximum data volume reduction while preserving the significant signal morphology features upon reconstruction. This has been achieved through the minimization of both the bit rate and the distortion of the reconstructed ECG signal through parameterization of the wavelet filters and the selection of optimum threshold levels of the wavelet coefficients in different subbands. 


\section{WAVELET PARAMETERIZATION}

The present study addresses the problem of finding a wavelet that best matches the wave shape of the ECG signal. In the framework of the proposed research methodology, an optimal wavelet can adequately represent a wavelet-compressed ECG signal at a given compression ratio. The optimality is detected by minimizing an error measure between the original signal and its compressed version, subject to the choice of wavelet. If, for a given wavelet, the error associated with the compressed signal is minimal, then its wavelet coefficients be considered to best represent the original signal. Therefore, the selected wavelet would more effectively match the signal under analysis when compared to standard wavelets [17]. In general, a square integrable function $x(t)$ can be represented in terms of translates and dilates of a single unique function $\psi(t)$ called the mother wavelet as

$$
f(t)=\sum_{j, n} c_{j, n} \psi_{j, n}(t)
$$

where, $\psi_{j, n}(t)=2^{j / 2} \psi\left(2^{j} t-n\right)$ are the set of orthogonal basis functions, obtained by dilating and translating $\psi(t)$ and $c_{j, n}$ are the DWT coefficients. Here, $j$ and, $n$ belong to the set of integers, $Z$ [17]. The DWT of the discrete type signal $x$ [n] of length $N$ is computed in a recursive cascade structure consisting of decimators $\downarrow 2$ and complementing low-pass $(h)$ and high-pass $(g)$ filters which are uniquely associated with a wavelet [8]. The signal is iteratively decomposed through a filter bank to obtain its discrete wavelet transform. This gives a new interpretation of the wavelet decomposition as splitting the signal into frequency bands. Figure (1) depicts a diagram of the filter bank structure. In hierarchical decomposition, the output from the low-pass filter constitutes the input to a new pair of filters. The filters coefficients corresponding to scaling (lowpass filter) and wavelet (highpass filter) functions are related by

$$
g[n]=(-1)^{n} h[L-n], n=0,1, \ldots, L-1
$$

where $L$ is the filter length. To adapt the mother wavelet to the signals for the purpose of compression, it is necessary to define a family of wavelets that depend on a set of parameters and a quality criterion for wavelet selection (i.e. wavelet parameter optimization). In this work, these concepts are adopted to derive a new approach for ECG signal compression based on dyadic discrete orthogonal wavelet bases, with selection of the mother wavelet leading to minimum reconstruction error. An orthogonal wavelet transform decomposes a signal in dilated and translated versions of the wavelet function $\psi(t)$. The wavelet function $\psi(t)$ is based on a scaling function $\phi(t)$ and both can be represented by dilated and translated versions of this scaling function.

$$
\phi(t)=\sum_{n=0}^{L-1} h(n) \phi(2 t-n) \quad \text { and } \quad \psi(t)=\sum_{n=0}^{L-1} g(n) \phi(2 t-n)
$$

With these coefficients $h(n)$ and $g(n)$ the transfer functions of the filter bank that are used to implement the discrete orthogonal wavelet transform can be formulated. 


$$
H(z)=\sum_{b=0}^{L-1} h(n) z^{-n} \quad \text { and } \quad G(z)=\sum_{b=0}^{L-1} g(n) z^{-n}
$$

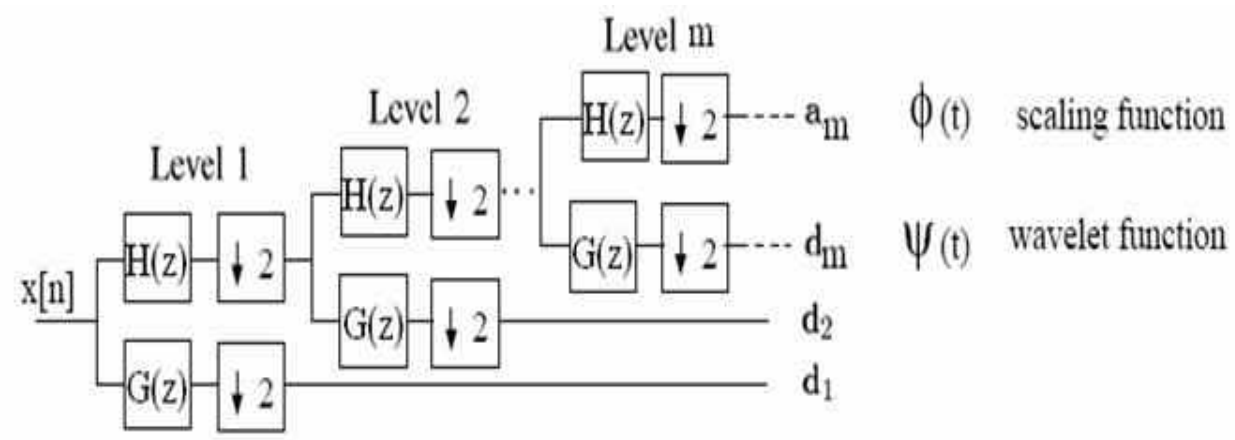

Forward Wavelet Transform

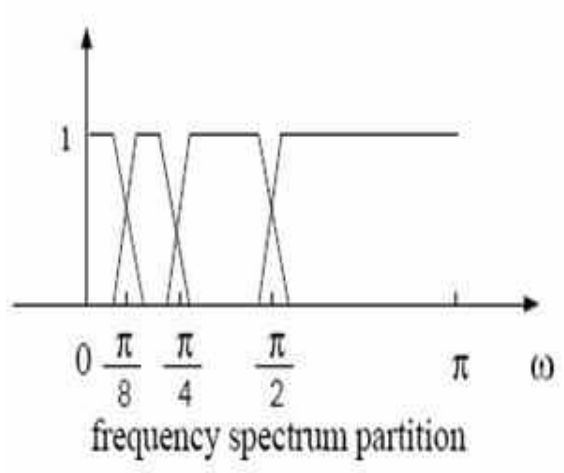

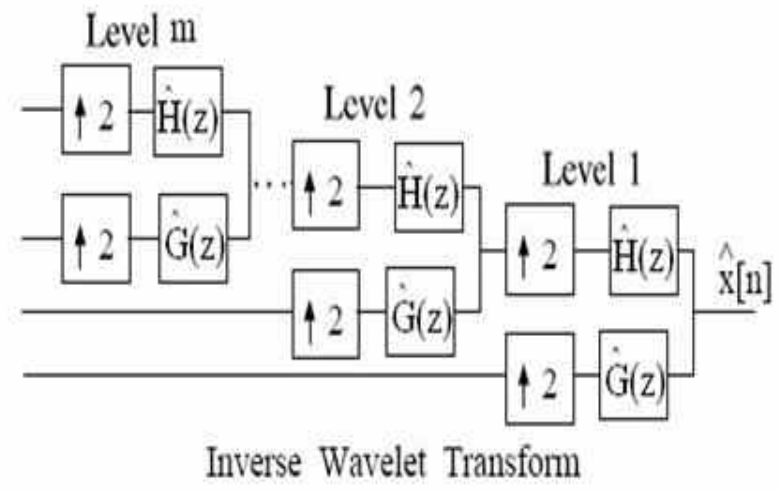

Inverse Wavelet Transform

Figure (1) The discrete dyadic wavelet transform as iteration of a 2-channel filter bank.

For a finite impulse response (FIR) filter of length $L$, there are $L / 2+1$ sufficient conditions to ensure the existence and orthogonality of the scaling function and wavelets [18]. Thus $L / 2-1$ degrees of freedom (free parameters) remain to design the filter $h$. The lattice parameterization described by Vaidyanathan [21] offers the opportunity to design $h$ via unconstrained optimization: the $L$ coefficients of $h$ can be expressed in term of $L / 2-1$ new free parameters. These parameters can be used to choose the wavelets which results in a good coding performance. The Daubechies wavelet family was constructed by using all the free parameters to maximize the number of vanishing moments. Coiflet wavelets were designed by imposing vanishing moments on both the scaling and wavelet functions. Wavelet parameterizations of Zou et al, [19] have been used to systematically generate $L$-tap orthogonal wavelets using the $L / 2-1$ free parameters for $L=4,6$ and 8 . The order of a wavelet filter is important in achieving good coding performance. A higher order filter can be designed to have good frequency localization which in turn increases 
the energy compaction. Consequently, by restriction to the orthogonal case, $h$ defines $\psi$. For this purpose consider, the orthogonal $2 \times 2$ rotational angles, realized by the lattice section shown in Figure (2), and defined by the matrix:

$$
R\left(\beta_{i}\right)=\left[\begin{array}{rr}
\cos \beta_{i} & -\sin \beta_{i} \\
\sin \beta_{i} & \cos \beta_{i}
\end{array}\right]
$$

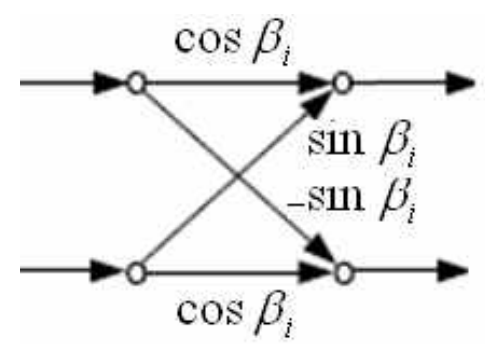

Figure (2) Lattice Implementation.

The polyphase matrix $H_{p}(z)$ can be defined in terms of the rotational angles as

$$
H_{p}(z)=\left[\begin{array}{ll}
H_{e}\left(z^{2}\right) & H_{o}\left(z^{2}\right) \\
G_{e}\left(z^{2}\right) & G_{o}\left(z^{2}\right)
\end{array}\right]=\prod_{i=o}^{L / 2-1}\left(\left[\begin{array}{cc}
\cos \beta_{i} & -\sin \beta_{i} \\
\sin \beta_{i} & \cos \beta_{i}
\end{array}\right]\left[\begin{array}{ll}
1 & 0 \\
0 & z^{-1}
\end{array}\right]\right)
$$

where, $H_{e}(z), H_{o}(z), G_{e}(z)$ and $G_{o}(z)$ are defined, respectively, from the decomposition of $H(z)$ and $G(z)$ as

$$
H(z)=H_{e}\left(z^{2}\right)+z^{-1} H_{o}\left(z^{2}\right)
$$

and $\quad G(z)=G_{e}\left(z^{2}\right)+z^{-1} G_{o}\left(z^{2}\right)$

To obtain the expressions for the coefficients of $H(z)$ in terms of the rotational angles, it is necessary to multiply out the above matrix product. In order to parameterize all orthogonal wavelet transforms leading to a simple implementation, the following facts should be considered.

1. Orthogonality is structurally imposed by using lattice filters consisting of orthogonal rotations.

2. The sufficient condition for constructing a wavelet transform, namely one vanishing moment of the wavelet, is guaranteed, by assuring the sum of all rotation angles of the of filters to be exactly $-45^{\circ}$.

A suitable architecture for the implementation of the orthogonal wavelet transforms are lattice filters. However, the wavelet function should be of zero mean, what is equivalent with the wavelet having at least one vanishing moment and the transfer functions $H(z)$ and $G(z)$ have at least one zero at $\mathrm{z}=-1$ and $\mathrm{z}=1$ respectively. These conditions are fulfilled if the sum of all rotation angles is $45^{\circ}$ [20], i.e., 


$$
\sum_{i=1}^{L / 2} \beta_{i}=45^{\circ}
$$

Therefore, a lattice filter whose sum of all rotation angles is $45^{\circ}$ performs an orthogonal WT independent of the angles of each rotation. For a lattice filter of length $L$, $L / 2$ orthogonal rotations are required. Denote the rotation angles by $\beta_{i}, i=1,2, \ldots, L / 2$, and considering the constraint given in (8), the number of design angles $\theta \mathrm{s}$ is $L / 2-1$. The following is the relation between the rotation angles and the design angles.

$$
\left.\begin{array}{l}
\beta_{1}=45^{\circ}-\theta_{1}, \\
\beta_{i}=(-1)^{i}\left(\theta_{i-1}+\theta_{i}\right) \text { for } i=2,3, \ldots, L / 2-1, \\
\beta_{L / 2}=(-1)^{L / 2} \theta_{L / 2-1}
\end{array}\right\}
$$

At the end of the decomposition process, a set of vectors representing the wavelet coefficients is obtained

$$
C=\left\{d_{1}, d_{2}, d_{3}, \ldots, d_{j}, \ldots, d_{m}, a_{m}\right\}
$$

where, $m$ is the number of decomposition levels of the DWT. This set of approximation and detail vectors represents the DWT coefficients of the original signal. Vectors $d_{j}$ contain the detail coefficients of the signal in each scale $j$. As $j$ varies from 1 to $m$, a finer or coarser detail coefficients vector is obtained. On the other hand, the vector $a_{m}$ contains the approximation wavelet coefficients of the signal at scale $m$. It should be noted that this recursive procedure can be iterated $\left(m \leq \log _{2} N\right)$ times at most. Depending on the choice of $m$, a different set of coefficients can be obtained. The inverse transform can be performed using a similar recursive approach. Thus, the process of decomposing the signal $x$ can be reversed, that is given the approximation and detail information it is possible to reconstruct $x$. This process can be realized as upsampling (by a factor of 2 ) followed by filtering the resulting signals and adding the result of the filters. The impulse responses $h$ ' and $g$ ' can be derived from $h$ and $g$. However, to generate an orthogonal wavelet, $h$ must satisfy some constraints. The basic condition is $\sum_{n=1}^{L} h(n)=\sqrt{2}$, to ensure the existence of $\phi$. Moreover, for orthogonality, $h$ must be of norm one and must satisfy the quadratic condition

$$
\sum_{n=1}^{L} h(n) \sum_{n=1}^{L} h(n-2 k)=0, \text { for } k=1, \ldots, L / 2-1
$$

The lattice parameterization described in [21] offers the opportunity to design $h$ using unconstrained optimization by expressing the $L / 2-1$ free parameters in terms of the design parameter vector $\theta$. For instance, if $L=6$, two-component design vector, $\theta=\left[\theta_{1}, \theta_{2}\right]$ is needed, and $h$ is given by [21]-[22]: 
$i=0,1 \quad h(i)=\left[\left(1+(-1)^{i} \cos \theta_{1}+\sin \theta_{1}\right)\left(1-(-1)^{i} \cos \theta_{2}-\sin \theta_{2}\right)+(-1)^{i} 2 \sin \theta_{2} \cos \theta_{1}\right] / 4 \sqrt{2}$

$i=2,3 \quad h(i)=\left[1+\cos \left(\theta_{1}-\theta_{2}\right)+(-1)^{i} \sin \left(\theta_{1}-\theta_{2}\right)\right] / 2 \sqrt{2}$

$i=4,5 \quad h(i)=1 / \sqrt{2}-h(i-4)-h(i-2)$

For other values of $L$, expressions of $h$ are given in [21]-[22]. With this wavelet parameterization there are infinite available wavelets which depend on the design parameter vector $\theta$ to represent the ECG signal at hand. Different values of $\theta$ may lead to different quality in the reconstructed signal. In order to choose the optimal $\theta$ values, and thus the optimal wavelet, a blind criterion of performance is needed. Figure (3) illustrates the block diagram of the proposed compression algorithm.

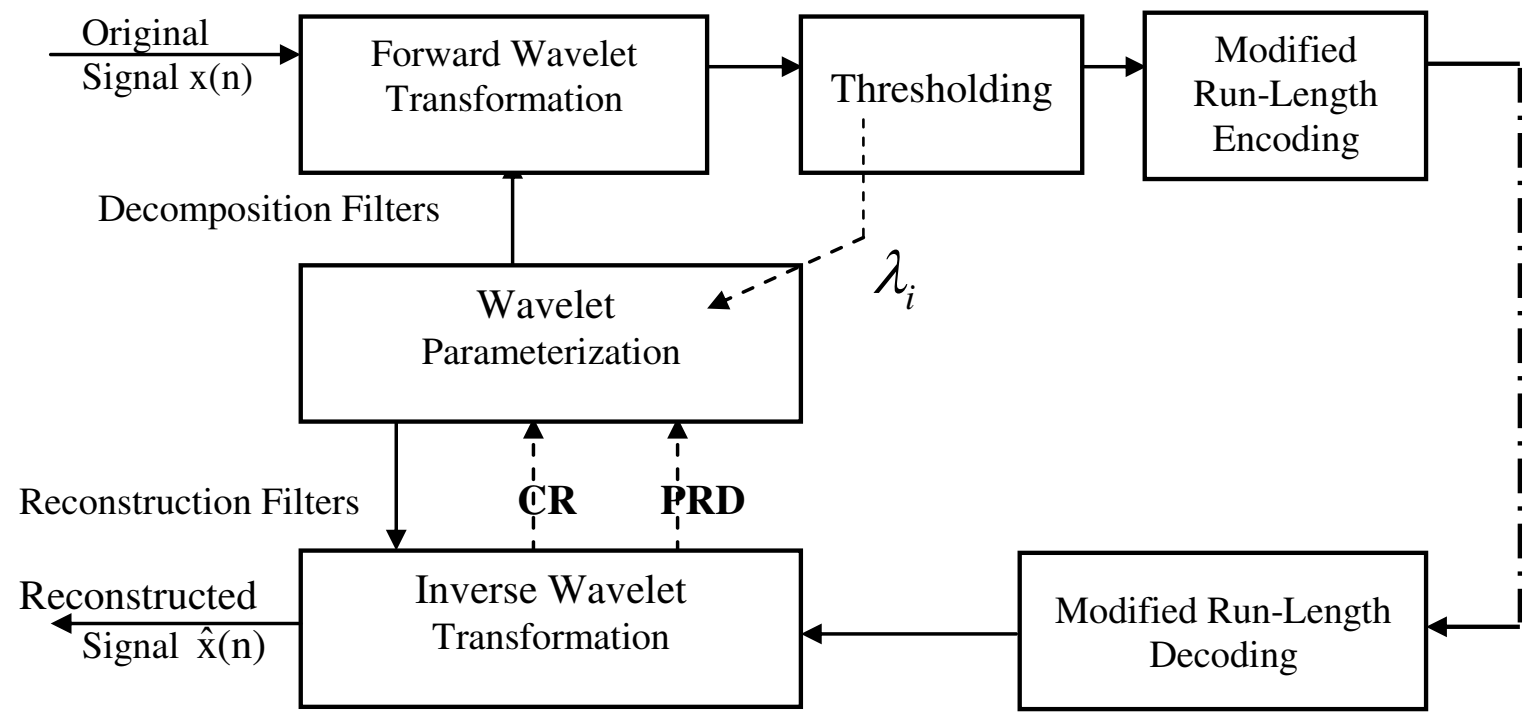

Figure (3) Block diagram for the proposed compression algorithm.

\section{THRESHOLD LEVELS SELECTION}

The main property of WT is that regions of little variation in original data manifest themselves as small or zero elements in the wavelet-transformed version. Hence, the WT of the signal contains a large number of detail coefficients, which are very small in magnitude. By fixing a nonnegative threshold, we can reset these small coefficients to zero resulting in a set of coefficients easier to store and transmit. Wavelet shrinkage and signal thresholding works in the following way [23]. When ECG signal is decomposed using wavelets, filters that act as averaging filters produce signal approximation and others produce details. If the details are small, they might be omitted without substantially affecting the main features of the data set. The idea of thresholding, then, is to set to zero all coefficients that are less than a particular threshold value. These coefficients are used in an inverse wavelet transformation to reconstruct the data set. A number of thresholding methods have been developed to 
allow the selection of the most pertinent components. In [24]-[22] concise descriptions of the commonly used wavelet thresholding methods together with a comprehensive list of references on the subject are given. Data compression methods require that only those wavelet coefficients which carry most of the signal information are identified and retained for use in the reconstruction of the signal.

Wavelet transform is energy invariant which means that total energy does not change when the wavelet transform is applied. This property indicates that near zero coefficients can be replaced by zeros without introducing too great distortion in the reconstructed signal. Energy compaction property of WT says that most of the energy of the signal is concentrated in fewer of the coefficients. Thus we need to keep coefficients with significant magnitudes only and kill the reset of the coefficients by considering them zeros. The elimination of small-valued coefficients can be brought about by setting all coefficients with value below a certain threshold equal to zero. Thresholds can be in general applied in two different modes, hard- and soft thresholding. In hard thresholding, coefficients whose absolute value does not exceed the threshold are zeroed, according to

$$
\hat{C}(i)= \begin{cases}0 & \text { if }|C(i)| \leq \lambda \\ C(i) & \text { otherwise }\end{cases}
$$

where, $\hat{C}(i)$ and $C(i)$ are the ith wavelet coefficient after and before thresholding respectively; and $\lambda$ is the threshold level. However, in soft thresholding the coefficients with absolute values below the threshold are zeroed and then the values of the nonzero remaining coefficients are shifted towards zero, according to

$$
\hat{C}(i)= \begin{cases}0 & \text { if }|C(i)| \leq \lambda \\ \operatorname{sign}(C(i))(|C(i)|-\lambda) & \text { if }|C(i)|>\lambda\end{cases}
$$

The amount of compression achieved and the distortion in the reconstructed signal can be controlled by choosing $\lambda$ values. In addition, the wavelet filters chosen should have high energy compaction properties. The main problem in ECG compression is the calculation of the threshold values that led to a predetermined reduction of wavelet coefficients. There are a variety of methods to choose the threshold level $\lambda$ in (13) or (14) for any given one-dimensional signal. Some methods proposed means for computing a better single threshold to apply to all the wavelet coefficients while others suggested using different thresholds for different subbands. These can be grouped into two categories: global thresholds and level-dependent thresholds. The former means that a single value of $\lambda$ is chosen to be applied globally to all empirical wavelet coefficients, while the latter means that a possibly different threshold value $\lambda_{\mathrm{j}}$ is chosen for each resolution level $\mathrm{j}$. In what follows, we consider the level-dependent thresholds. These thresholds all require an estimate of the noise level $\sigma$. The usual standard deviation of the data values is clearly not a good estimator. Donoho and Johnstone, considered estimating $\sigma$ in the wavelet domain and suggested a robust estimate that is based only on the empirical wavelet coefficients at the finest resolution level. The reason for considering only the finest level is that corresponding empirical wavelet coefficients tend to consist mostly of noise. Since there is some 
signal present even at this level, they proposed a robust estimate of the noise level $\sigma$ based on the median absolute deviation [27].

\section{MODIFIED RUN-LENGTH CODING}

The wavelet domain representation itself does not introduce any compression. Compression is obtained by encoding the thresholded wavelet coefficients using optimal thresholding levels. Given that most of the energy in the signal is in the lower subbands, it is reasonable to assume that after thresholding a substantial number of higher band wavelet coefficients will be set to zeros. Since these zeros tend to occur in clusters, as a direct consequence of the way in which the data are organized in vectors, run-length coding of these zeros makes sense. The basic idea of this technique is to encode a sequence of equal symbols with a certain codeword depending on the length of that sequence. Thus, two types of codewords follow each other alternatively is used: the counter-words and the value-words. For example, the string "aaabbbbd" is encoded as: $(\mathrm{a}, 3),(\mathrm{b}, 4)$, and $(\mathrm{d}, 1)$. In case of ECG compression, the run-length coding is done by representing the thresholded wavelet coefficients vectors in the forum of (Run, Level), where Run is the number of zeros before each nonzero coefficients, and Level is the amplitude of the coefficient following a number of zeros given by Run. The event that the last coefficients are all zeros is represented by the special code $(0,0)$. For example, the set of wavelet coefficients given by $W_{\text {before }}=\left[\begin{array}{llllllllll}0 & 1 & 0 & 0 & 0 & 4 & 5 & 0 & 0 & 0\end{array}\right.$

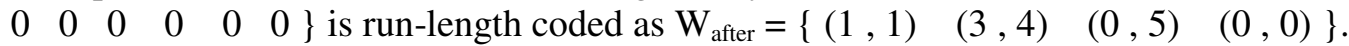
As it has been mentioned in section 3 , the compression is based on representing the thereshold wavelet coefficients with a small number of bits. This has been carried out by discarding the WT-coefficients, which are less than a given threshold. These coefficients are considered insignificant with their values set to zero. The remaining $\mathrm{N}_{\mathrm{S}}$ coefficients are the significant coefficients. The number of the discarded coefficients is $\mathrm{N}_{\mathrm{I}}=\mathrm{N}-\mathrm{N}_{\mathrm{S}}$. Most of these coefficients are concentrated at the end of the coefficients' vector. In technical literature, many algorithms are suggested to deal with signals with repeated samples' values such as run-length coding and Huffman coding. The need of at least one bit for the mostly repeated sample is the main limitation of the Huffman coding. The disadvantage of the run-length algorithm is the need of two words for the representation of each group of repeated samples: one for the repeated value and the other for the number of repetitions.

In this section more efficient coding algorithm, modified run-length algorithm, is introduced for dealing with this situation. The algorithm is based on representing each significant coefficient by $b_{S}+1$ bits. The insignificant coefficients (of value zero) are manipulated in a different manner. First, the repeated groups of zeros are counted and the resulting count is represented by $b_{S}+1$ bits. Then the train of coefficients representing the ECG signal is transformed to another train of numbers. Some of these numbers represent the significant coefficients and the rest are the numbers representing the repeated group of zeros $\left(K_{1}, K_{2}, \ldots, K_{M}\right)$. Here, $M$ denotes the number of these groups. The problem here is how to differentiate between the coefficients and the numbers representing the group of zeros. For example, the number 18 may be found twice in the new train of numbers, where the first 18 may be a significant coefficient and the second one may indicate 18 repeated zeros. To come over this problem, the 
first bit in the representation of each number is used as a control bit. In case of the significant coefficient this bit is set to one and in case of repeated zeros it is reset to zero.

_ representation of significant residual coefficient

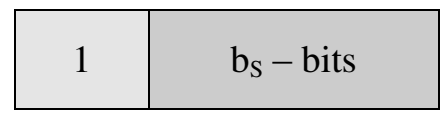

- representation of a group of repeated zeros

\begin{tabular}{|l|l|}
\hline 0 & $\mathrm{~b}_{\mathrm{s}}-\mathrm{bits}$ \\
\hline
\end{tabular}

\section{PERFORMANCE MEASURES}

The criteria for testing the performance of the compression algorithms consist of three components: compression measure, reconstruction error and computational complexity. The compression measure and the reconstruction error depend usually on each other and determine the rate-distortion function of the algorithm. The computational complexity component is related to practical implementation consideration and is desired to be as low as possible. The compression ratio $(C R)$ is defined as the ratio of the number of bits representing the original signal to the number required for representing the compressed signal. So, it can be calculated from:

$$
C R=\frac{N b_{c}}{\left(N_{S}+M\right)\left(b_{S}+1\right)}
$$

Where, $b_{c}$ is the number of bits representing each ECG sample. One of the most difficult problems in ECG compression applications and reconstruction is defining the error criterion. Several techniques exist for evaluating the quality of compression algorithms. The most obvious way to determine the preservation of diagnostic information is to subject the reconstructed data to evaluation by a cardiologist. This approach might be accurate in some cases but suffers from many disadvantages. One drawback is that it is a subjective measure of the quality of reconstructed data and depending on the cardiologist being consulted, different results may be presented. Another shortcoming of the approach is that it is highly inefficient. Moreover, the subjective judgment solution is expensive and can generally be applied only for research purposes [8]. The distortion resulting from the ECG processing is objectively measured by the percent root-mean-square difference $(P R D)$ [3]. However, in previous trials focus has been on how much compression a specific algorithm can achieve without loosing too much diagnostic information. In most ECG compression algorithms, the $P R D$ measure is employed. It is defined as

$$
P R D=\sqrt{\frac{\sum_{n=1}^{N}(x(n)-x(n))^{2}}{\sum_{n=1}^{N} x^{2}(n)}}
$$

This error estimate is the one most commonly used in all scientific literature concerned with ECG compression techniques. The clinical acceptability of the reconstructed 
signal is desired to be as high as possible. The main drawbacks are the inability to cope with baseline fluctuations and the inability to discriminate between the diagnostic portions of an ECG curve. However, its simplicity and relative accuracy make it a popular error estimate among researchers. As the PRD is heavily dependent on the mean value, it is more appropriate to use the modified criteria:

$$
P R D_{1}=\sqrt{\frac{\sum_{n=1}^{N}(x(n)-x(n))^{2}}{\sum_{n=1}^{N}(x(n)-\bar{x})^{2}}}
$$

where, $\bar{x}$ is the mean value of the signal. Furthermore, it is established in [3], that if the $P R D_{1}$ value is between 0 and $9 \%$, the quality of the reconstructed signal is either 'very good' or 'good', whereas if the value is greater than $9 \%$ its quality group cannot be determined. As we are strictly interested in very good and good reconstructions, it is taken that the $P R D_{l}$ value, as measured with (17), must be less than $9 \%$. In this paper, the $P R D_{1}$ error measure has been chosen. The decision was based upon the fact that it is a rather simple measure requiring few non-complex calculations. Also, it is currently the prime error estimate used in almost all literature concerning ECG compression.

\section{PROPOSED OPTIMIZATION-BASED COMPRESSION ALGORITHM}

As it has been mentioned before, many of the resulting wavelet coefficients are either zero or close to zero. These coefficients are divided into two classes according to their energy contents; namely: high energy coefficients and low energy coefficients. By coding only the larger coefficients, many bits are already discarded. The high energy coefficients should be compressed very accurately because they contain more information. So, they are threshold with low threshold levels. However, the low energy coefficients that represent the details are threshold with high threshold levels. The wavelet selection step involves choosing an analysis wavelet and allocating bits to each coefficient in the resulting wavelet representation. The success of this scheme is based on the fact that only a fraction of nonzero value wavelet coefficients may be encoded using a small number of bits. The main idea behind the proposed approach is to find the minimum distortion representation of a signal, subject to a given bit budget or to find the minimum bit rate representation of a signal, subject to a target $P R D$.

In order to establish an efficient solution scheme, the following precise problem formulation is developed. For this purpose, consider the one-dimensional vector $x(i), i=1,2,3, \ldots ., N$ represents the frame of the ECG signal to be compressed; where $N$ is the number of its samples. The initial threshold values are selected as follows. The threshold value is computed separately for each subband by finding the mean $(\mu)$ and standard deviation $(\sigma)$ of the absolute of the non-zero wavelet coefficients in the corresponding subband. If the $\sigma$ is greater than $\mu$ then the threshold value in that subband is set to $(2 * \mu)$, otherwise, it is set to $(\mu-\sigma)$. Also, define the 
targeted performance measures $P R D_{\text {targte }}$ and $C R_{\text {target }}$ and start with an initial wavelet design parameter vector $\theta=\left[\theta_{10}, \theta_{20}, . . ., \theta_{L-10}\right]$ to construct the wavelet filters $H(z)$ and $G(z)$ adopting equation (12).

1) Use designed parameters to construct the wavelet decomposition filters to decompose the ECG signal into its equivalent $m$ subbands represented by the vector $Y(\theta)=\left[Y_{1}, Y_{2}, Y_{3}, \ldots, Y_{m}\right]$.

2) Threshold the obtained wavelet coefficients with threshold levels $\lambda_{i}, i=1,2,3$,

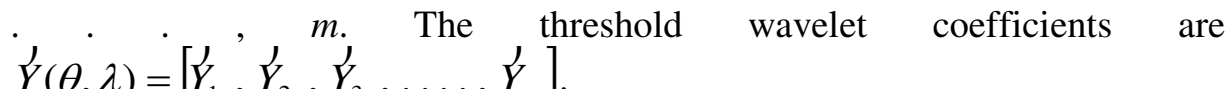

3) Use the modified run-length coding algorithm to represent the threshold coefficients with minimum storage capacity.

4) Decode the stored coefficients and reconstruct the signal using the reconstruction filters deduced from the wavelet filters' parameters.

5) Calculate the PRD $\left(\mathrm{PRD}_{1}\right)$ using equation 16 (17) and check the acceptance of the signal quality using $P R D \leq \mathrm{PRD}_{\text {targte }}\left(\mathrm{PRD}_{1} \leq \mathrm{PRD}_{1 \text { targte }}\right)$. If $\mathrm{PRD}>\mathrm{PRD}_{\text {targte }}\left(\mathrm{PRD}_{1}>\mathrm{PRD}_{1 \text { targte }}\right)$, modify the threshold levels, based on energy contents of the subands' representations and go to step (2); otherwise go to step (6).

6)

min imize $\sqrt{\frac{\sum_{n=1}^{N}(x(n)-x(n))^{2}}{\sum_{n=1}^{N}(x(n))^{2}}}$ subject that $P R D \leq P R D_{t \arg e t}$

minimize $\sqrt{\frac{\sum_{n=1}^{N}(x(n)-x(n))^{2}}{\sum_{n=1}^{N}(x(n)-\bar{x})^{2}}}$ subject that $P R D_{1} \leq P R D_{1_{t} \text { arget }}$

7) Calculate the $\mathrm{CR}$ and check the acceptance of the bit rate using $\mathrm{C} R \geq \mathrm{C} R_{\text {targte }}$. If the compression ratio is less than $\mathrm{C} R_{\text {targte }}$, modify the wavelet design parameter vector $\theta=\left[\theta_{1}, \theta_{2}, \ldots, \theta_{L-1}\right]$ and go to step (2); otherwise go to step (7).

minimize $\left(\frac{1}{C R}\right)=\frac{\text { number of bits of the compressed signal } \underset{x}{\mathrm{\sigma}}(n)}{\text { number of bits of the original signal } x(n)}$

subject that $C R \geq C R_{\text {target }}$

8) end. 
where, $\left\{(n)=W T^{-1}\{Y(\theta, \lambda)\}\right.$ is the reconstructed signal, and $W T^{-1}\{$.$\} is the inverse$ wavelet transformation. The problem is that the wavelet coefficients corresponding to the QRS regions are higher than those in the other subbands. Thus direct minimization of (17) results in minimum distortion in least mean square sense; however the visual investigation of the reconstructed signal is not satisfactory. This problem has been solved by introducing a weighting vector $W=\left[W_{1}, W_{2}, W_{3}, \ldots, W_{m}\right]$; where $W_{i}$ is high for small coefficients subands and is low for high coefficients subands. Taking into consideration the weighting process, the reconstructed signal is calculated as $\left\{(n)=W T^{-1}\left\{W^{*} Y\right\}\right.$. Keeping those (potentially) important coefficients, the distortion error is reduced and signal estimation is improved. Equations 18 (19) and 20 are solved by adopting the Matlab function fminimax. In all cases considered, the solution is obtained within 12 iterations. Figure (4) illustrates the compression algorithm for satisfying predefined $C R$ and $P R D\left(P R D_{1}\right)$.

\section{RESULTS AND DISCUSSION}

Common criteria for performance testing are the compression ratio $\mathrm{CR}$ and the percentage root-mean-square difference $\mathrm{PRD}_{1}$ (independent from the mean value), defined as in section 5. The values of $\mathrm{PRD}_{1}$ between $0 \%$ and $9 \%$ guarantee good reconstructed signals. Here, the reconstructed signals have been evaluated using the percentage root mean square difference $P R D\left(P R D_{l}\right)$ defined by equation $16(17)$ and the compression ratio defined by equation (15) are used as quantitative performance measures. The MIT-BIH Arrhythmia database [1], which has been extensively used to evaluate ECG compression methods, has been used for performance evaluation of the proposed algorithm. We have selected a subset of 2 records (117, and 119) of 4 min duration. We will refer to them simply as the database. The sampling frequency is 360 $\mathrm{Hz}$ and the resolution is $11 \mathrm{bps}$. It should be noted that the performance of the compression algorithm depends on the record being compressed. Before running the compression algorithm, the wavelet transform parameters and the threshold levels in different subbands should be initially selected. To select the decomposition and reconstruction filters, we have to seek a good trade-off between compression performance and computational complexity. Generally compression performance is better for longer filters, and computational complexity is smaller for the shorter filters. The tested signals have been decomposed up to the fifth decomposition level by utilizing six parameters orthogonal wavelet filters. For this case, the parameterization consists of three orthogonal rotations: $\beta_{1}=-45^{\circ}-\theta_{1}, \beta_{2}=\left(\theta_{1}+\theta_{2}\right)$ and $\beta_{3}=-\theta_{2}$. Thus, the pair of design angles $\left(\theta_{1}, \theta_{2}\right)$ defines completely the case of $L=6$. The minimization process starts by considering $\theta_{1}=22.6^{\circ}$ and $\theta_{2}=6.03^{\circ}$ as initial design angles. In fact, these values correspond to the Daubechies' wavelet of length $L=6$. The initial threshold values are selected as explained in section 6 . Now, considering the compression of records 117 and 119 with the target CR and PRD given as: $C R_{t \arg e t}=23, \quad P R D_{t \arg e t} \leq 1.6 \%$ for record 117 and $C R_{t \arg e t}=25$, $P R D_{t \arg e t} \leq 2.2 \%$ for record 119 , solutions are obtained after 8 and 10 iterations 
respectively. In the two cases, the wavelet parameters and the threshold levels are optimized. Compressing the same two signals with the standard six parameters Daubechies and Coiflet orthogonal wavelet filters and allowing only the threshold levels in subbands to vary, the distortion in the reconstructed signal is higher than that obtained with optimized filters. The parameters of a Coifet filter are $\theta_{1}=-122.85^{\circ}$ and $\theta_{2}=192.15^{\circ}$. Table (1) summarizes the comparison between the performance of the proposed signal dependent wavelet parameterization method and Daubechies and Coiflet standard wavelets. From the obtained results, it can be deduced that the optimized wavelet gives highest $C R$ and lowest $P R D$. However, the computational complexity of the proposed technique is the price paid for the improvement in the performance measures. Table (2) illustrates the parameters of the wavelet filters used in getting the results given in Table (1).

Table (1): Comparison between the performance of the proposed signal dependent wavelet parameterization method and other standard wavelets when the threshold levels are optimized in all subbands

\begin{tabular}{|c|c|c|c|c|}
\hline \multirow{2}{*}{$\begin{array}{l}\text { MIT-BIH } \\
\text { Record }\end{array}$} & \multirow{2}{*}{ Wavelet filters } & \multicolumn{2}{|c|}{ Performance Measures } & \multirow{2}{*}{$\begin{array}{l}\text { Computation Cost } \\
\text { (number of } \\
\text { iterations) }\end{array}$} \\
\hline & & $\mathrm{PRD}_{1}(\%)$ & $\mathrm{CR}$ & \\
\hline 117 & \multirow{2}{*}{ Optimized wavelet filters } & 1.6 & $23.0: 1$ & 8 \\
\hline 119 & & 2.2 & $25.0: 1$ & 10 \\
\hline 117 & \multirow{2}{*}{ Coiflet wavelet filters } & 5.2 & $16.5: 1$ & 1 \\
\hline 119 & & 4.7 & $18.1: 1$ & 1 \\
\hline 117 & \multirow{2}{*}{ Daubechies wavelet filters } & 7.1 & $8.0: 1$ & 1 \\
\hline 119 & & 6.7 & $10.1: 1$ & 1 \\
\hline
\end{tabular}

Table (2) Rotational angles and wavelet filters' parameters used in getting the results given in Table (1).

\begin{tabular}{|c|c|c|c|c|c|c|c|c|c|c|}
\hline \multirow{2}{*}{$\begin{array}{c}\text { MIT- } \\
\text { BIH } \\
\text { Record }\end{array}$} & \multirow{2}{*}{$\begin{array}{c}\text { Wavelet } \\
\text { filters }\end{array}$} & \multicolumn{2}{|c|}{ Rotational angles } & \multicolumn{6}{|c|}{ Wavelet filters' parameters } \\
\cline { 3 - 10 } & $\beta_{1}$ & $\beta_{2}$ & $\beta_{3}$ & $h_{0}$ & $h_{1}$ & $h_{2}$ & $h_{3}$ & $h_{4}$ & $h_{5}$ \\
\hline 117 & $\begin{array}{c}\text { Optimized } \\
\text { wavelet } \\
\text { filters }\end{array}$ & 3.1367 & 1.1921 & -3.5434 & -0.0007 & -0.1446 & 0.3675 & 0.8534 & 0.3403 & -0.0017 \\
\cline { 4 - 11 } & 3.2763 & 1.2444 & -3.7354 & 0.0241 & -0.1778 & 0.4197 & 0.8492 & 0.2633 & 0.0357 \\
\hline 119 & $\begin{array}{c}\text { Coiflet } \\
\text { wavelet } \\
\text { filter }\end{array}$ & 2.9295 & 1.2095 & -3.3537 & -0.0157 & -0.0727 & 0.3849 & 0.8526 & 0.3379 & -0.0727 \\
\hline 117 & $\begin{array}{c}\text { Daubechies } \\
\text { wavelet } \\
\text { filter }\end{array}$ & 0.3910 & 0.4997 & -0.1052 & 0.0352 & -0.0854 & -0.1350 & 0.4599 & 0.8069 & 0.3327 \\
\hline 119 & & & & & & & & & &
\end{tabular}




\section{Original ECG Signal}

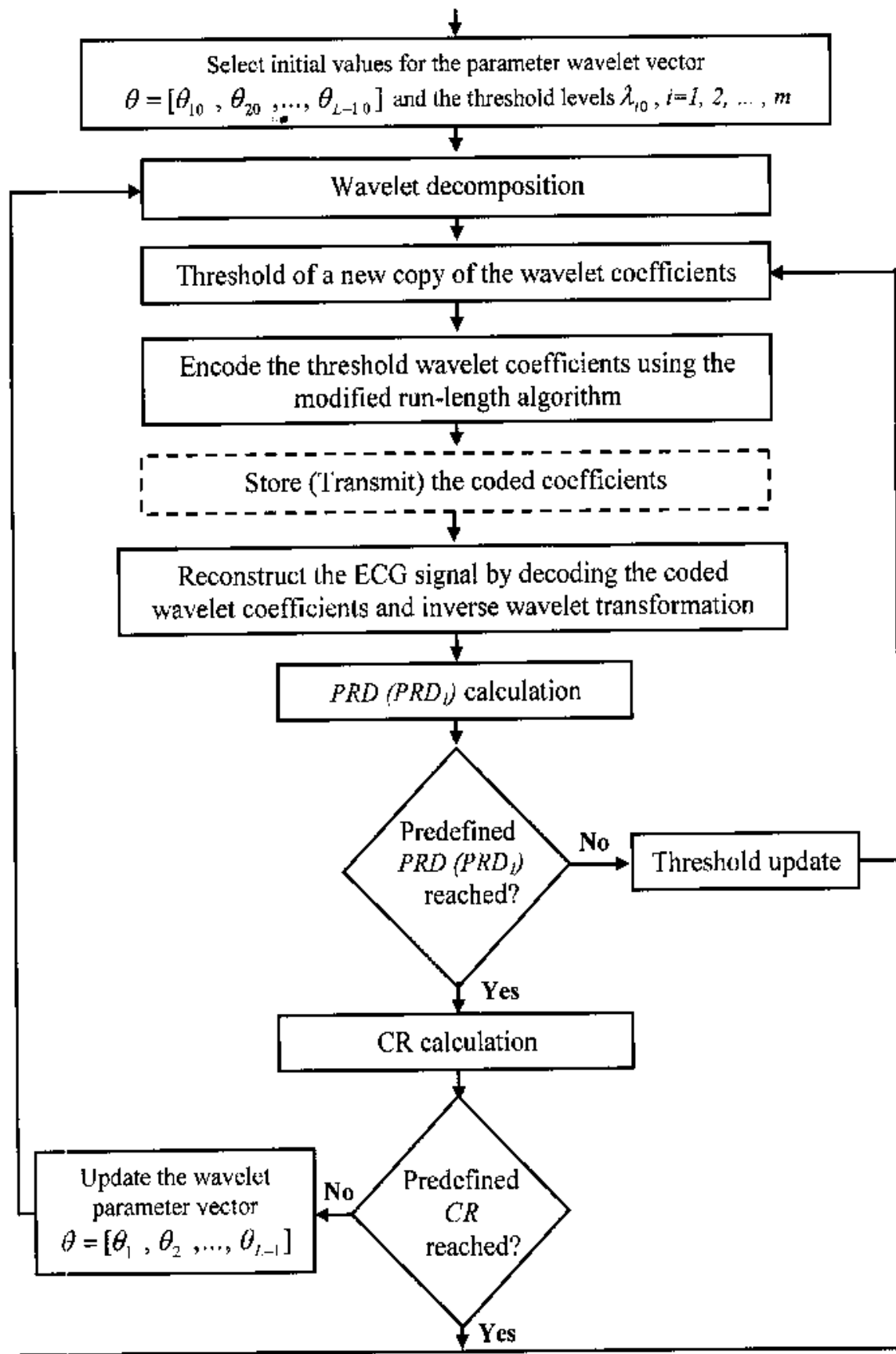

Parameters for mother wavelet filter $\theta_{o p t}=\left[0_{\text {lopt }}, \theta_{2 o p t}, \ldots, \theta_{L-1 \text { opt }}\right]$ and the threshold levels $\lambda_{\text {iopt }} i=1,2, \ldots, J$

\section{End}

Figure (4) Compression Algorithm for Satisfying Predefined the $C R$ and $\operatorname{PRD}\left(P R D_{I}\right)$. 


\section{CONCLUSION}

Wavelet bases may be better suited than other transformations; however one mother wavelet may be better than another for a specific signal or in different experimental sessions. In most cases, it is not possible to choose a prior the best wavelet, which also depends on the encoding scheme. So, wavelet parameterization is necessary. In the context of signal compression, a natural optimization criterion is the mean square error of the compressed signal with respect to the original signal. It was shown that within the same ECG signals, the best basis functions may be largely different for different CRs, and with significant differences in the reconstruction error. This paper introduces a new DWT based technique for the compression of ECG signals with minimum loss of diagnostics information. It is based on the parameterization of wavelet filters that can offer better timefrequency localization and the optimal selection of threshold levels in different sub-bands that yields to efficient coding of the wavelet coefficients. The main feature of the proposed ECG compression algorithm is the achievement of information rate reduction, while retaining the relevant diagnostic information in the reconstructed signal. Experimental results obtained by running the compressor on records 117 and 119 shows that the proposed method is capable of achieving good $C R$ values with low distortion and at the same time provide preservation of the shapes and amplitudes of the important ECG waves. The obtained results show superiority of the proposed method at all target PRD values. From these results, it can be deduced that the performance of the optimized signal dependent wavelet parameterization outperforms that of Daubechies and Coiflet standard wavelets. However, the computational complexity of the proposed technique is the price paid for the improvement in the compression performance measures. Finally, it should be noted that the proposed method is flexible in controlling the quality of the reconstructed signals and the volume of the compressed signals by establishing target PRD and CR a prior respectively.

\section{REFERENCES}

[1] G. Moody. MIT-BIH Arrhythmia Database, Massachusetts Institute of Technology, Cambridge, Aug. $1992 . \quad$ Available Online: http://www.physionet.org/physiobank/database/mitdb/

[2] D. Haugland, J. Heber, and J. Husøy, "Optimization algorithms for ECG data compression,” Med. Biol. Eng. Comput, vol. 35, pp. 420-424, July 1997.

[3] Sabah M. Ahmed, A. Al-Shrouf and M. Abo-Zahhad, "ECG data compression using optimal non-orthogonal wavelet transform," Med. Eng. Phys., vol. 22, pp. 39-46, 2000.

[4] Miaou S-G and Lin C-L "A quality-on-demand algorithm for wavelet-based compression of electrocardiogram signals" IEEE Trans. Biomed. Eng. 49 233-9, 2002.

[5] A. Al-Shrouf, M. Abo-Zahhad and Sabah, M. A., "A Novel Compression Algorithm for Electrocardiogram Signals Based on the Linear Prediction of the Wavelet Coefficients" International Journal on Digital Signal Processing, vol. 12, pp. 604-622, 2003. 
[6] Sabah M.A., "ECG Data Compression Algorithm Based on the Combination of Singular Value Decomposition and Discrete Wavelet Transform" Journal of Engineering Sciences, Assiut University, pp. 2267-2280, November 2005.

[7] Sabah M.A., "ECG Signal Compression Using Combined Modified DiscreteCosine And Discrete-Wavelet Transforms" Journal of Engineering Sciences, Assiut University, Vol. 34, No. 1, pp. 215-226, Jan. 2006.

[8] S. Jalaleddine, C. Hutchens, R. Strattan and W. Coberly, "ECG data compression techniques-a unified approach," IEEE Trans. Biomed. Eng., vol. 37, pp. 329-343, 1990.

[9] P. S Addison, "Wavelet transforms and the ECG a review," Topical Review, Institute Of Physics Publishing, Physiological Measurement, vol. 26, pp. R155R199, Aug. 2005.

[10] M. M Elena, J. M Quero, I. Borrego, "An optimal technique for ECG noise reduction in real time applications," Computers in Cardiology, vol. 33, pp. 225-228, 2006.

[11] M. del Mar Elena, J. M. Quero, and I. Borrego, "Optimal selection of wavelet coefficients for electrocardiograph compression," ETRI Journal, vol. 29, no. 4, pp. 530-532, Aug. 2007.

[12] L. Brechet, M. F. Lucas, C. Doncarli, and D. Farina, "Compression of biomedical signals with mother wavelet optimization and best-basis wavelet packet selection," IEEE Trans. On Biomedical Engineering, Vol. 54, no. 12, pp. 2186-2192, Dec. 2007.

[13] M. Nielsen, E. N. Kamavuako, M. M. Andersen, M. F. Lucas, and D. Farina, "Optimal wavelets for biomedical signal compression," Med. Biol. Eng. Comput., vol. 44, pp. 561-568, 2006.

[14] A. Antoniadis, "Wavelet methods in statistics some recent developments and their applications," Statistics Surveys, vol. 1 pp. 16-55, Dec. 2007.

[15] Benzid, R., Marir, F., Boussaad, A., Benyoucef, M., and Arar, D., "Fixed percentage of wavelet coefficients to be zeroed for ECG compression," Electronics Letters, vol. 39, no. 11, pp. 830-831, 2003.

[16] M. Blanco-Velasco, F. Cruz-Rolda'n, J. I. Godino-Llorente and K. E. Barner, "ECG compression with retrieved quality guaranteed," Electronics Letters, vol. 40, no. 23, pp. 900-901, 2004.

[17] I. Daubechies, "Orthonormal bases of compactly supported wavelets", Communications on Pure and Applied Mathematics, vol. 41, No. 7, pp. 909-996, Nov. 1998.

[18] D. L. Donoho and I. M. Johnstone, "Minimax estimation via wavelet shrinkage," Ann. Statist., vol. 26, pp. 879-921, 1998.

[19] H. Zou and A. H. Tewfik, "Parameterization of compactly supported orthonormal wavelets," IEEE Trans. on Signal Processing, vol. 41, no. 3, pp. 1428-1431, March 1993.

[20] H. Xie and J. M. Morris, "Design of orthonormal wavelets with better timefrequency resolution," Proc. Of SPIE, Wavelet Applications, pp. 878-997, Orlando, Florida, 1994.

[21] P.P. Vaidyanathan, "Multirate digital filters, filter banks, polyphase networks and applications: A tutorial review", Proceedings of the IEEE, vol. 41, No. 12, pp. 3463-3479, Dec. 1993. 
[22] A. Maitrot, M. F. Lucas, C. Doncarli, and D. Farina, "Signal-dependent wavelet for electromyogram classification," Med. Biol. Eng. Comput., vol. 43, pp. 48792, 2005.

[23] D. L. Donoho, "De-noising by soft-thresholding," IEEE Trans. on Information Theory, vol. 41, no. 3, pp. 613-627., 1995

[24] S. A. Chouakri, F. Bereksi-Reguig, "Wavelet denoising of the electrocardiogram signal based on the corrupted noise estimation," Computers in Cardiology, vol. 32, pp. 1021-1024, 2005.

[25] S. Poornachandral and N. Kumaravel, "Subband adaptive shrinkage for denoising of ECG signals," EURASIP Journal on Applied Signal Processing, vol. Article ID 81236, pp. 1-9, 2006.

[26] B. N. Singh and A. K. Tiwari, "Optimal selection of wavelet basis function applied to ECG signal denoising," Digital Signal Processing, vol. 16, pp. 275287, 2006.

[27] M. Kania, M. Fereniec, R. Maniewski, "Wavelet Denoising for Multi-lead High Resolution ECG Signals," Measurement Science Review, vol. 7, No. 4, pp. 3033, 2007.

\section{الاختيار الأمثل لمستويات الددود ومرشحات المويجة لضغط أشارة القلب بجودة عالية د. صباح محمد أحمد}

\section{أستاذ مساعد بقسم الهندسة الكهربائية ـ كلية الهندسة - جامعة أسيوط}

بالرغم من ان معظم الأمور النظرية والتطبيقية لخوارزميات ضغط أثنارة النشاط الكهربائي للقلب المبنية على محول المويجة قد تمت دراستها بطريقة جيدة الا انه مازال هناك بعض المسائل المتعلقة باختيار

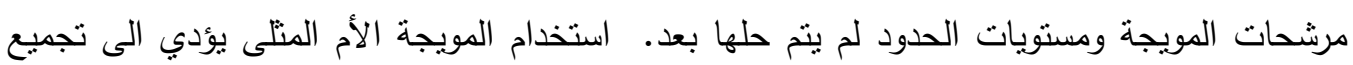
معاملات المويجة في نطاق مكاني واحد والى زيادة الحد الأعلى لقيم المعاملات في النطاق المويجي.

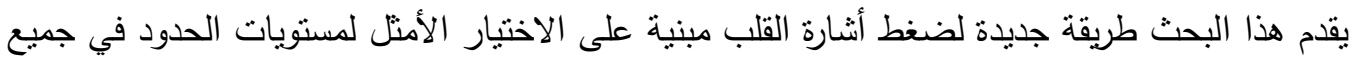

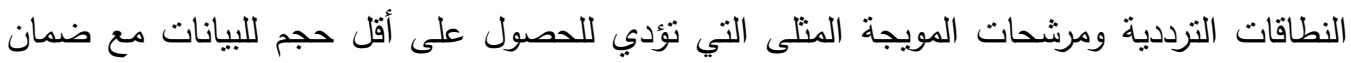
كفاءة استرجاع الإثارة. الطريقة المقترحة تبدأ بتقسيم الإثارة الي إطارات frames ينت تحليلها بواسطة

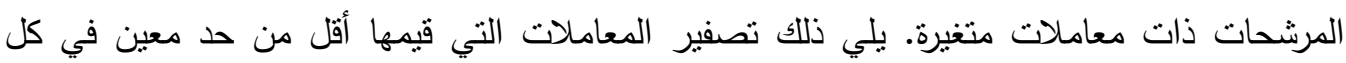
نطاق ترددي والإبقاء على المعاملات الأخرى كما هي ثم استخدام طريق تكويد التكرار -الطول المعدلة modified run-length coding بطريقة مثالية بحيث يتم ضغط الإثارة الي نسبة الضغط المطلوبة وبالدقة المطلوبة. ولقد نم اختبار

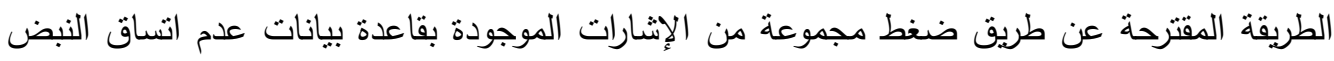

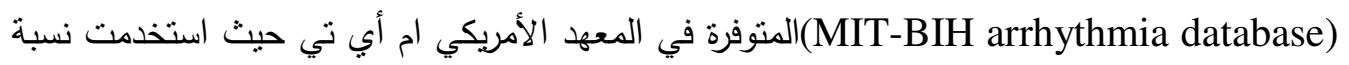


الضغط (CR) و النسبة المئوية للجذر التربيعي لمتوسط مربع الخطأ (PRD) والجذر التربيعي لمتوسط الفرق بين مربع الخطأ ومربع المتوسط (

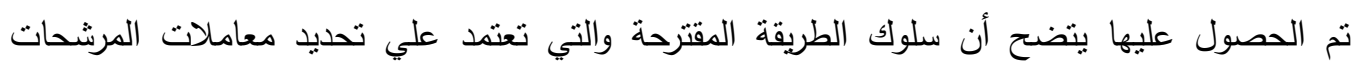
المستخدمة بطريقة مثالية وفقا للإثارة المراد ضغطها من حيث نسبة الضغط والدقة في استرجاع الإثارة تفوق تلك التي استخدمت فيها مرشحات دبوتشيز Daubechies وكوافلت Coiflet القياسية. ويؤخذ على هذه الطريقة ان الثمن المدفوع للتحسين في مقاييس سلوك عملية الضغط هو زيادة كمية الحسابات

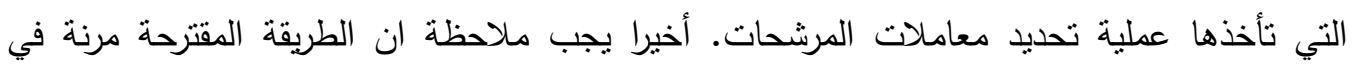

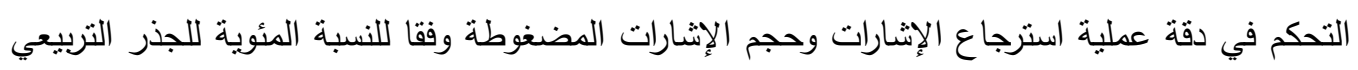
لمتوسط مربع الخطأ و نسبة الضغط المحددة مسبقا. 